\title{
Nonmonotonic composition dependence of vibrational phase relaxation rate in binary mixtures
}

\author{
Swapan Roychowdhury and Biman Bagchi ${ }^{\text {) }}$ \\ Solid State and Structural Chemistry Unit, Indian Institute of Science, Bangalore 560 012, India
}

\begin{abstract}
We present here isothermal-isobaric $N-P-T$ ensemble molecular dynamics simulations of vibrational phase relaxation in a model system to explore the unusual features arising due to concentration fluctuations which are absent in one component systems. The model studied consider strong attractive interaction between the dissimilar species to discourage phase separation. The model reproduces the experimentally observed nonmonotonic, nearly symmetric, composition dependence of the dephasing rate. In addition, several other experimentally observed features, such as the maximum of the frequency modulation correlation time $\tau_{c}$ at mole fraction near 0.5 and the maximum rate enhancement by a factor of about 3 above the pure component value, are also reproduced. The product of mean square frequency modulation $\left[\left\langle\Delta \omega^{2}(0)\right\rangle\right]$ with $\tau_{c}$ indicates that the present model is in the intermediate regime of inhomogeneous broadening. The nonmonotonic composition $\chi_{A}$ dependence of the dephasing time $\tau_{v}$ is found to be primarily due to the nonmonotonic $\chi$ dependence of $\tau_{c}$, rather than due to a similar dependence in the amplitude of $\left\langle\Delta \omega^{2}(0)\right\rangle$. The probability distribution of $\Delta \omega$ shows a markedly non-Gaussian behavior at intermediate composition $\left(\chi_{A} \simeq 0.5\right)$. We have also calculated the composition dependence of the viscosity in order to explore the correlation between the composition dependence of viscosity $\eta^{*}$ with that of $\tau_{v}$ and $\tau_{c}$. It is found that both the correlation time essentially follow the composition dependence of the viscosity. A mode coupling theory is presented to include the effects of composition fluctuations in binary mixture.
\end{abstract}

\section{INTRODUCTION}

The study of vibrational phase relaxation (VPR) has been an important endeavor of a physical chemist/chemical physicist in the attempt to understand and quantify the interaction of a chemical bond with the surrounding solvent molecules in liquids and solids. ${ }^{1-4}$ When studied in conjunction with different experimental techniques such as NMR, ESR, vibrational infrared (IR) and Raman spectroscopy, and electronic excitation, theoretical models for line shapes have provided useful information and insight. In the above experimental techniques, the experimental setup is made in such a way that an ensemble of molecules is probed. The simultaneous homogeneous and inhomogeneous line broadening due to the time dependence and the variance of the environments at different reference molecules contribute to the observed line shape. The situation is more complicated in binary liquid mixtures, because different molecules see different concentrations of species in the immediate surrounding such that they contribute to the spectrum at different frequencies. The effect of concentrations has been explored for a variety of systems with infrared and Raman spectroscopy. ${ }^{5,6}$ The nonmonotonic, but nearly symmetric, composition dependence of VPR rate in binary mixtures has been a subject of long standing interest in chemical physics/ physical chemistry.

\footnotetext{
${ }^{a)}$ Author to whom correspondence should be addressed. Electronic mail: bbagchi@sscu.iisc.ernet.in
}

The problem of vibrational dephasing in liquid mixtures has attracted considerable amount of attention in the past two decades. ${ }^{5,7-10}$ In condensed phase, a reference molecule interacts with the neighboring molecules and the observed spectra contain information regarding the intramolecular as well as intermolecular interactions. A precise determination of the isotropic Raman linewidths and the band positions, using a $90 \AA$ scattering geometry may allow, in favorable cases, a systematic investigation of the dephasing mechanisms in binary liquid mixtures. ${ }^{7}$ The time domain coherent anti-Stokes Raman spectroscopy (CARS) technique has been used to study the frequency modulation correlation time $\tau_{c}$ in the $\mathrm{C}-\mathrm{I}$ stretch in the binary liquid mixture $\left(\mathrm{CH}_{3} \mathrm{I}\right.$ and $\mathrm{CDCl}_{3}$ ) by Laubereau and co-workers (see Fig. 3 in Ref. 5) Measurements of the concentration dependence allow separating the dephasing contribution by concentration fluctuations. The time constant of dephasing was determined to be in the range of $1-3 \mathrm{ps}$ in the temperature range $242 \mathrm{~K}-374 \mathrm{~K}$. The linewidth of the $\nu_{1}$ band displayed a anomalous concentration dependence with a maximum at a mole fraction of $\chi=0.5$, in both the IR and Raman studies. Bondarev and Mardaeva ${ }^{11}$ explained this dependence in terms of additional line broadening due to slow concentration fluctuations assuming a stationary concentration distribution of Gaussian shape in a microscopic volume around the reference molecule. This assumption, based on Anderson and Kubo theory, leads to a symmetric linewidth dependence around $\chi=0.5$ which, although common, has not been ob- 
served in every example. Knapp and Fisher ${ }^{8,9}$ included the dynamical aspect into the theory, i.e., concentration fluctuation due to diffusion, in order to explain a more general type of concentration and line broadening. This simple yet elegant theory has been successful in explaining several aspects of dephasing observed in experiments. Muller et al. ${ }^{6}$ recently reported an evidence for the spectral diffusion in Raman echo of $\mathrm{C}-\mathrm{I}$ stretch in the $50 \%$ mixture of $\mathrm{CH}_{3} \mathrm{I}$ and $\mathrm{CDCl}_{3}$. From a comparison with CARS and conventional Raman data, an inhomogeneous broadening contribution was deduced with a lifetime of $4-7 \mathrm{ps}$ at room temperature and was assigned to concentration fluctuations. However, the existing theories are mostly phenomenological.

The objective of this paper is to develop a description which remedies the shortcomings of the early theoretical treatments and to provide a quantitative explanation of the above nonmonotonic composition dependence. Note that not only the static but also the dynamic aspects of the concentration fluctuations due to the influence of translational and rotational motion need to be considered on equal footing. Therefore, we have included the relative contribution of vibration-rotation coupling on the total linewidth. It turns out that this contribution cannot be ignored.

Vibrational dephasing rate in liquid is known to show a strong dependence on viscosity. ${ }^{1}$ In fact, hydrodynamic calculation shows that the dephasing rate is proportional to the viscosity of the medium. ${ }^{1}$ It is particularly interesting in the present context because the viscosity of a binary mixture often shows nonmonotonic composition dependence. ${ }^{12} \mathrm{We}$ have carried out a detailed analysis of the dependence between the viscosity and the dephasing rate.

It was shown earlier that when properly formulated, mode coupling theory can provide quite accurate description of the dephasing rate. ${ }^{13}$ Binary mixture provides a particularly challenging situation for mode coupling theory because composition fluctuation is a slow process which should be within the purview of the mode coupling theory description. We present a formulation of the mode coupling theory and present qualitative analysis.

The present simulations reproduce the nonmonotonic composition dependence of the dephasing rate. The calculated features agree closely with the experimental observations. In addition, both the simulation and the mode coupling theory analysis show that it is the concentration fluctuation which is mostly responsible for the unusual features observed in the composition dependence of Raman linewidth. Interestingly, the composition dependence of the dephasing rate is found to be closely related to the composition dependence of the viscosity. Although this close resemblance might be due to the specific features of the model employed, it suggests that experiments should compare the two dependencies.

The organization of the rest of the paper as follows. In the following section, we present the theoretical background and the main equations. In Sec. III, we present both the simulation details and the model of the system used in this study. Section IV contains the results and discussions. A mode coupling theory analysis is given in Sec. V. We close the paper with a few concluding remarks in Sec. VI.

\section{THEORETICAL BACKGROUND}

The theories of the vibrational dephasing are usually based on Kubo's stochastic theory of the line shape. ${ }^{1-3}$ This gives the following simple expression for the isotropic Raman line shape $[I(\omega)]$ in terms of frequency fluctuation time correlation $\left[C_{\omega}(t)\right]$ :

$$
I(\omega)=\int_{0}^{\infty} d t e^{(i \omega t)}\left[\operatorname{Re} e^{\left(i \omega_{0} t+i(\Delta \omega) t\right)} e^{\left[-\int_{0}^{t} d t^{\prime}\left(t-t^{\prime}\right)\left\langle\Delta \omega\left(t^{\prime}\right) \Delta \omega(0)\right\rangle\right]}\right],
$$

where $\Delta \omega_{i}(t)=\omega_{i}(t)-\left\langle\omega_{i}\right\rangle$ is the fluctuation of the vibrational frequency from average vibrational frequency and $\omega_{0}$ is the fundamental vibrational frequency whose dephasing is studied. Three terms, (a) density $\left[\Delta \omega_{\rho}(t)\right]$, (b) vibration-rotation (VR) coupling $\left[\Delta \omega_{V R}(t)\right]$, and (c) resonance $\left[\Delta \omega_{R s}(t)\right]$ are responsible for the vibrational frequency fluctuations. Autocorrelations and cross-correlations between atom-atom forces, VR coupling, and resonance terms have been considered in our model. The average relaxation time or dephasing time is defined by

$$
\tau_{v}=\int_{0}^{\infty} C_{Q}(t) d t
$$

where $C_{Q}(t)=\langle Q(t) Q(0)\rangle$ and $Q(t)$ is a time dependent normal coordinate whose dephasing is being studied. It is a normalized coordinate.

The average frequency correlation decay time is given by

$$
\tau_{c}=\int_{0}^{\infty}\left[C_{\omega}(t) / C_{\omega}(0)\right] d t,
$$

where $C_{\omega}(t)=\langle\Delta \omega(t) \Delta \omega(0)\rangle$ is the frequency modulation time correlation function.

In the Markovian limit, $\tau_{c}$ and $\tau_{v}$ are related by ${ }^{1,3}$

$$
1 / \tau_{v}=\left\langle\Delta \omega^{2}(0)\right\rangle \tau_{c} .
$$

Thus, the essential quantity is the frequency modulation time correlation function $C_{\omega}(t)$ which also describes spectral diffusion.

The frequency modulation time correlation function is dependent on the frequency and the harmonic and anharmonic force constants of the vibrational mode whose dephasing is considered. The Hamiltonian for the vibrational mode of the diatomic is assumed to be of the following usual form: ${ }^{1}$

$$
\begin{aligned}
H_{v i b} & =1 / 2 \mu \omega_{0}^{2} x^{2}+1 / 6 f x^{3} \\
& =K_{11} Q^{2}+K_{111} Q^{3},
\end{aligned}
$$

where $\mu$ is the reduced mass of the diatom, $\omega_{0}$ is the vibrational frequency, and $Q=\mu^{1 / 2} x$ is the normal coordinate. $K_{11}$ $\left(1 / 2 \omega_{0}^{2}\right)$ and $K_{111}$ are the harmonic and anharmonic force constants respectively. We have taken the value of $\omega_{0}$ is $525 \mathrm{~cm}^{-1}$. The value of $K_{111}\left(=f / 6 \mu^{3 / 2}\right)$ is taken to be $1.72 \mathrm{~g}^{-1 / 2} \mathrm{~cm}^{-1} \mathrm{~s}^{-2}$ which is the value for the C-I stretch. The notation used here is taken from Oxtoby. ${ }^{1}$ The total Hamil- 


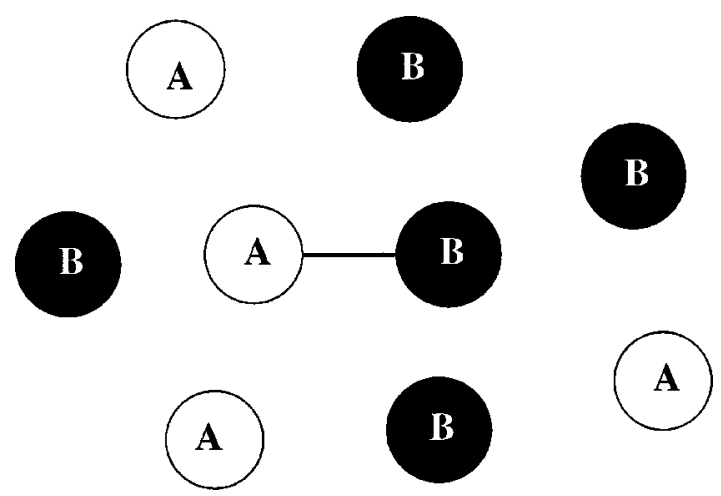

FIG. 1. The schematic diagram illustrating the model of our heteronuclear diatom $(A-B)$ in the binary mixture consisting of atoms $A$ and $B$.

tonian of the system consists of $H_{v i b}$, the bath Hamiltonian and the interaction part.

\section{SYSTEM AND SIMULATION DETAILS}

We have constructed a simple model of binary mixture to probe effects of composition fluctuation. We consider VPR of a heteronuclear diatom $A-B$ (modeled after $\mathrm{CH}_{3} \mathrm{I}$ ). We have taken $A-B$ diatom in a mixture of $A$ (modeled after $\mathrm{CH}_{3}$ - a pseudosphere) and $B$ (modeled after iodine atomic sphere) (see Fig. 1). An isothermal-isobaric ensemble $(N-P-T)$ simulation study of composition fluctuation of model binary Lennard-Jones (LJ) is presented. The LJ parameters for the $A$ system were taken as $\epsilon_{A} / k_{B}=146.46 \mathrm{~K}$ and $\sigma_{A}=3.85 \AA$ and, for the $B$ as $\epsilon_{B} / k_{B}=400 \mathrm{~K}$ and $\sigma_{B}$ $=4 \AA$. The LJ parameters of $A B$ intermolecular interaction are taken as $\epsilon_{A B} / k_{B}=467 \mathrm{~K}$ and $\sigma_{A B}=4.6 \AA^{13}{ }^{13}$ Simulations in the $N-P-T$ ensemble are performed using the Nose-HooverAnderson method, ${ }^{14-17}$ where the external reduced temperature is held fixed at $T^{*}=1.0$, the external pressure has been kept at $P^{*}=2$ and the total number of particle $(N)$ is 512 of which 40 are diatoms. The integration step is $0.0002 \tau$

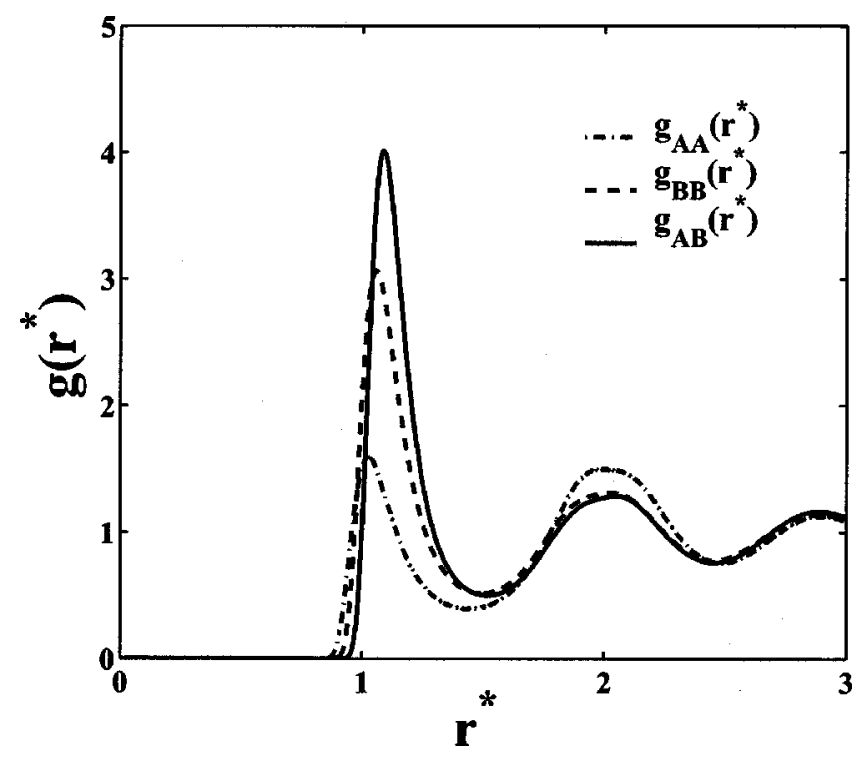

FIG. 2. Partial radial distribution functions, $g_{A A}\left(r^{*}\right)$ (solute-solute), $g_{B B}\left(r^{*}\right)$ (solvent-solvent), and $g_{A B}\left(r^{*}\right)$ (solute-solvent) at 0.5 solute composition $\left(\chi_{A}\right) \cdot r^{*}=r / \sigma_{A}$, where $\sigma_{A}$ is the molecular diameter of species $A$.
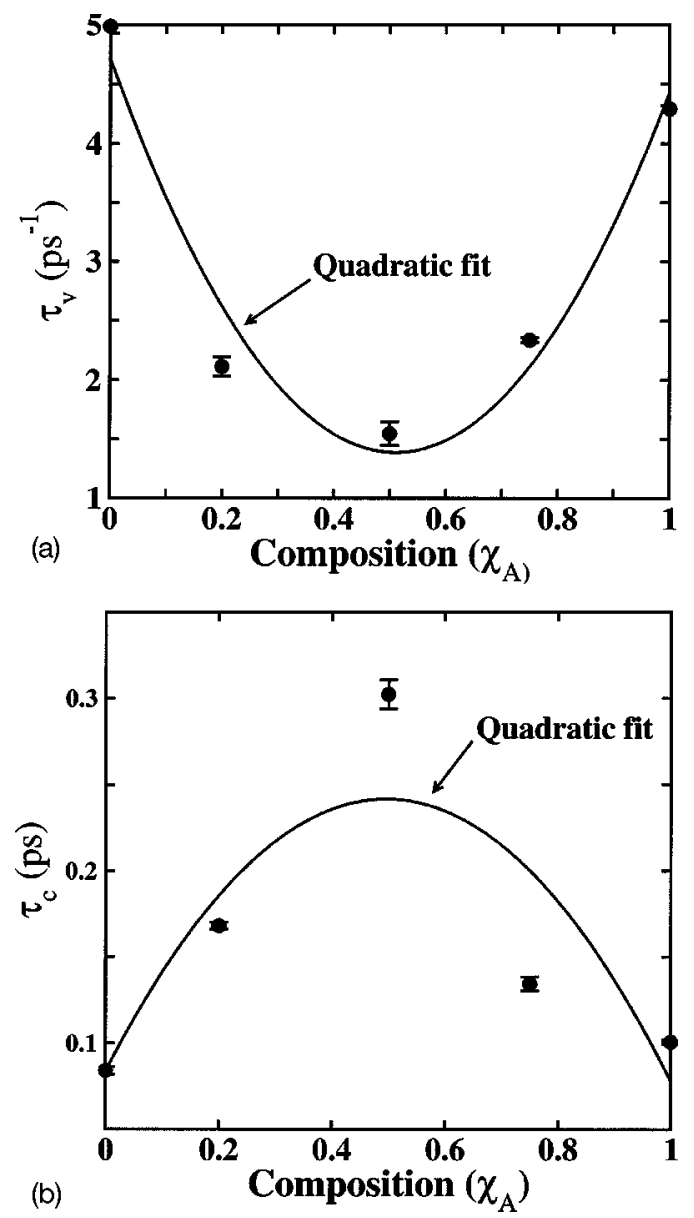

FIG. 3. (a) The calculated average dephasing time $\tau_{v}$ and (b) the average correlation time $\tau_{c}$ of $A-B$ bond at different mole fractions $\left(\chi_{A}\right)$. We also show the fit through the data as a guide to eye.

$\left(=\sqrt{m \sigma^{2} / \epsilon}\right)$. We have simulated the system for five different concentrations. Each simulation consist of $10^{5}$ equilibration steps, followed by $3 \times 10^{5}$ production steps during which time averaging has been performed. In addition, at each composition, four to five of such long runs have been employed to improve the statistics and also to obtain the error bars. Such extensive averaging was necessary to include the effects of slow composition fluctuation.

While our model is clearly oversimplified, it is sufficiently simple to permit detailed theoretical analysis. As it retains the essential feature of a binary mixture, it can be expected to describe many aspects of vibrational dephasing in binary mixtures, as is indeed the case, shown below.

For intermolecular potential-energy $V_{i j}$ between two molecules $i$ and $j(A$ and $B$ ), the following site-site LennardJones type is employed as given below

$$
V_{i j}=\sum_{\alpha, \beta}^{1,2} V\left(r_{i \alpha j \beta}\right) .
$$

Here $V\left(r_{i \alpha j \beta}\right)$ is the Lennard-Jones atom-atom potential defined as 

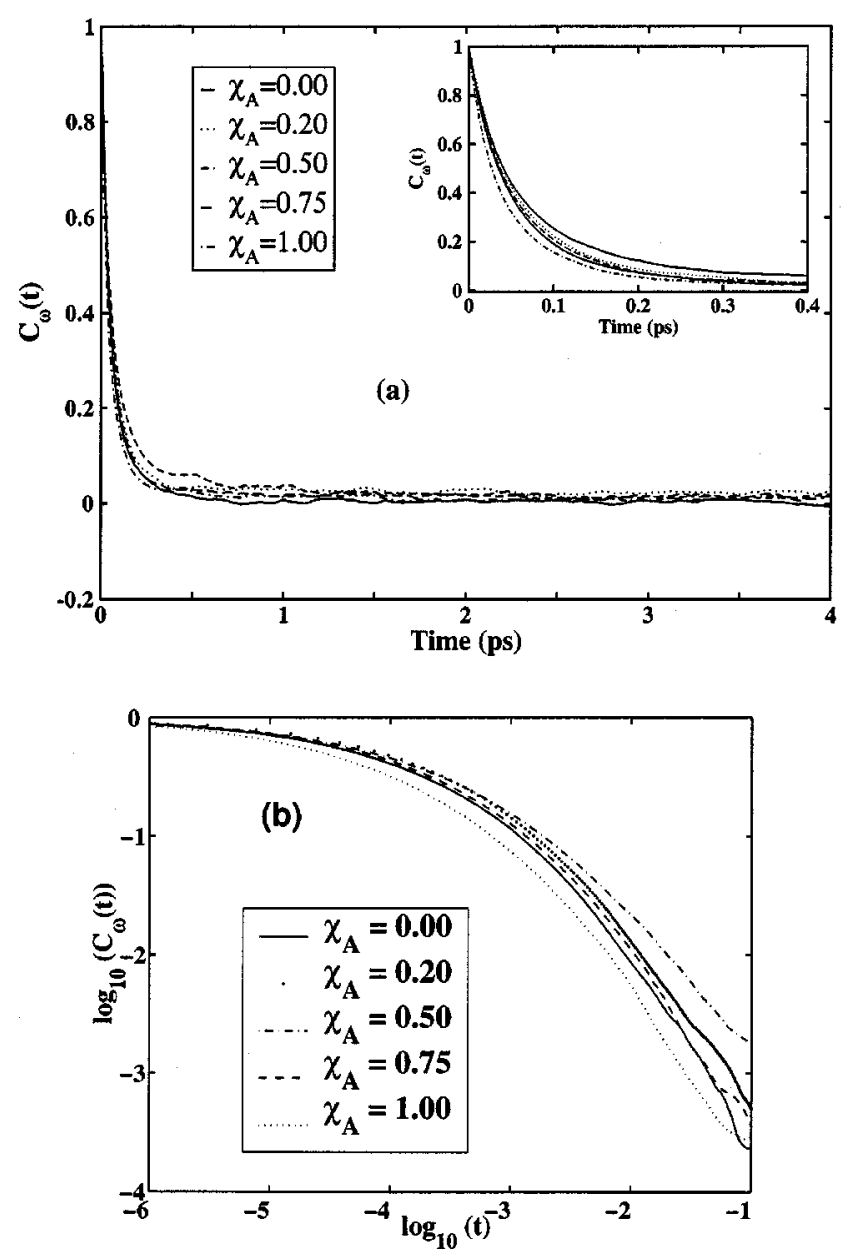

FIG. 4. (a) The frequency modulation time correlation functions $\left[C_{\omega}(t)\right]$ at different compositions of the solute $\left(\chi_{A}\right)$. Inset show the same up to $0.4 \mathrm{ps}$. In (b) we have plotted $\log _{10}\left[C_{\omega}(t)\right]$ vs $\log _{10}(t)$ at all five compositions.

$$
V\left(r_{i \alpha j \beta}\right)=4 \epsilon_{i \alpha j \beta}\left[\left(\frac{\sigma_{i \alpha j \beta}}{r_{i \alpha j \beta}}\right)^{12}-\left(\frac{\sigma_{i \alpha j \beta}}{r_{i \alpha j \beta}}\right)^{6}\right] .
$$

Vibrational coordinate dependence of $\epsilon$ and $\sigma$ has been incorporated according to Everitt and Skinner. ${ }^{18}$ We have followed their scheme to do the same.

\section{RESULTS AND DISCUSSION}

In Fig. 2, we plot the partial radial distribution functions $g_{i j}\left(r^{*}\right)\left(r^{*}=r / \sigma_{A}\right)$ obtained from the simulation of our model. As the solute-solvent interaction strength affects the structure surrounding a solute/solvent molecule to a great extent, ${ }^{19}$ the above observed features are reflected in the increment of correlation among the unlike species, $g_{A B}\left(r^{*}\right)$, for 0.5 mole fraction. We can refer to such behavior as the structure forming, or preferential solvation.

The calculated results for the dephasing parameters are plotted in Fig. 3(a) and 3(b). It is interesting to notice the shortening of the dephasing time $\tau_{v}$ with decreasing concentration from the neat liquid of $A, \chi_{A}=1.0$, to $\chi_{A} \simeq 0.5$ and the subsequent rise again with further dilution. The average correlation time $\tau_{c}$ simultaneously increases from a value around $0.5 \mathrm{ps}$ to $\simeq 0.25 \mathrm{ps}$ and drops again. These results are comparable to known experimental results. ${ }^{5}$ The changes in-

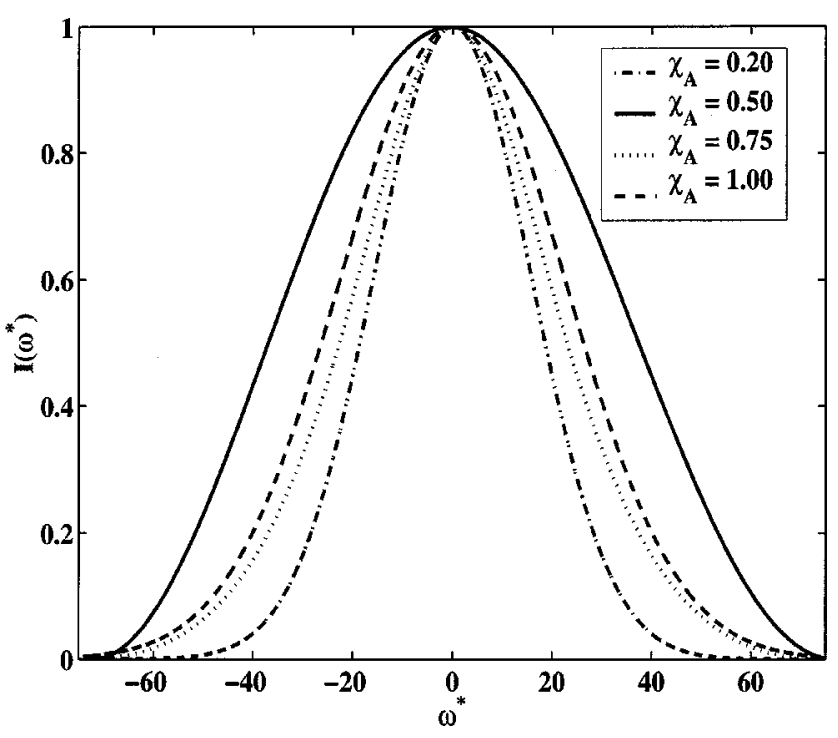

FIG. 5. The frequency dependent Raman line shape at four different compositions in the binary mixture. Note that the frequency is in unit of $\omega_{0}$, the fundamental frequency.

dicate a strong contribution of concentration fluctuations to the phase relaxation with a remarkably slow time scale of the process. As expected for mixture with notably larger and smaller concentrations, the mechanism approaches the homogeneous limit. Most attempts to move a solvent molecule either into or out of the first solvation shell create a conflict with the positions of other molecules. Thus, molecular exchange is a slow process. This mechanism is absent in a neat liquid.

In Fig. 4(a), the frequency modulation time correlation function $C_{\omega}(t)$, is plotted against time $t$ at five different compositions of $A\left(\chi_{A}\right)$. Note that the decay of $C_{\omega}(t)$ is essentially complete within 4 ps or so. Much of the correlation decays with an ultrafast time constant. Thus, $C_{\omega}(t)$ decays to about 0.2 (that is, $80 \%$ ) by $100 \mathrm{fs}$. This is followed by a slow decay which is most noticeable for the composition $\chi_{A}=0.5$. In Fig. 4(b) we have plotted $\log _{10}\left[C_{\omega}(t)\right]\left[C_{\omega}(t)\right]$ versus $\log _{10}\left[C_{\omega}(t)\right](t)$ at all five compositions. This figure clearly shows the slow decay at the said composition $\left(\chi_{A}=0.5\right)$. This slow decay is responsible for the increase in dephasing rate at the intermediate composition. This slow decay is due to composition (or concentration) fluctuation, as was discussed by Knapp and Fisher. ${ }^{8,9}$

In Fig. 5, we show the calculated vibrational (Raman) line shape. It is seen that two different kinds of behavior are present in the line shape. The line shape shows a nearly Gaussian behavior at all compositions except at the composition $\chi_{A}=0.5$ where it looks more like a non-Gaussian. The line shape reflects the distribution of frequency modulation at different compositions.

In order to understand the sharp increase in width of the line shape on approaching to equimolar mole fraction, we have computed the probability distribution of frequency fluctuations at different compositions (see in Fig. 6). For compositions away from the equimolar distribution of species, the probability distribution $[P(\Delta \omega)]$ is approximately Gaussian. However, at the equimolar distribution $\left(\chi_{A} \simeq 0.5\right) P(\Delta \omega)$ 

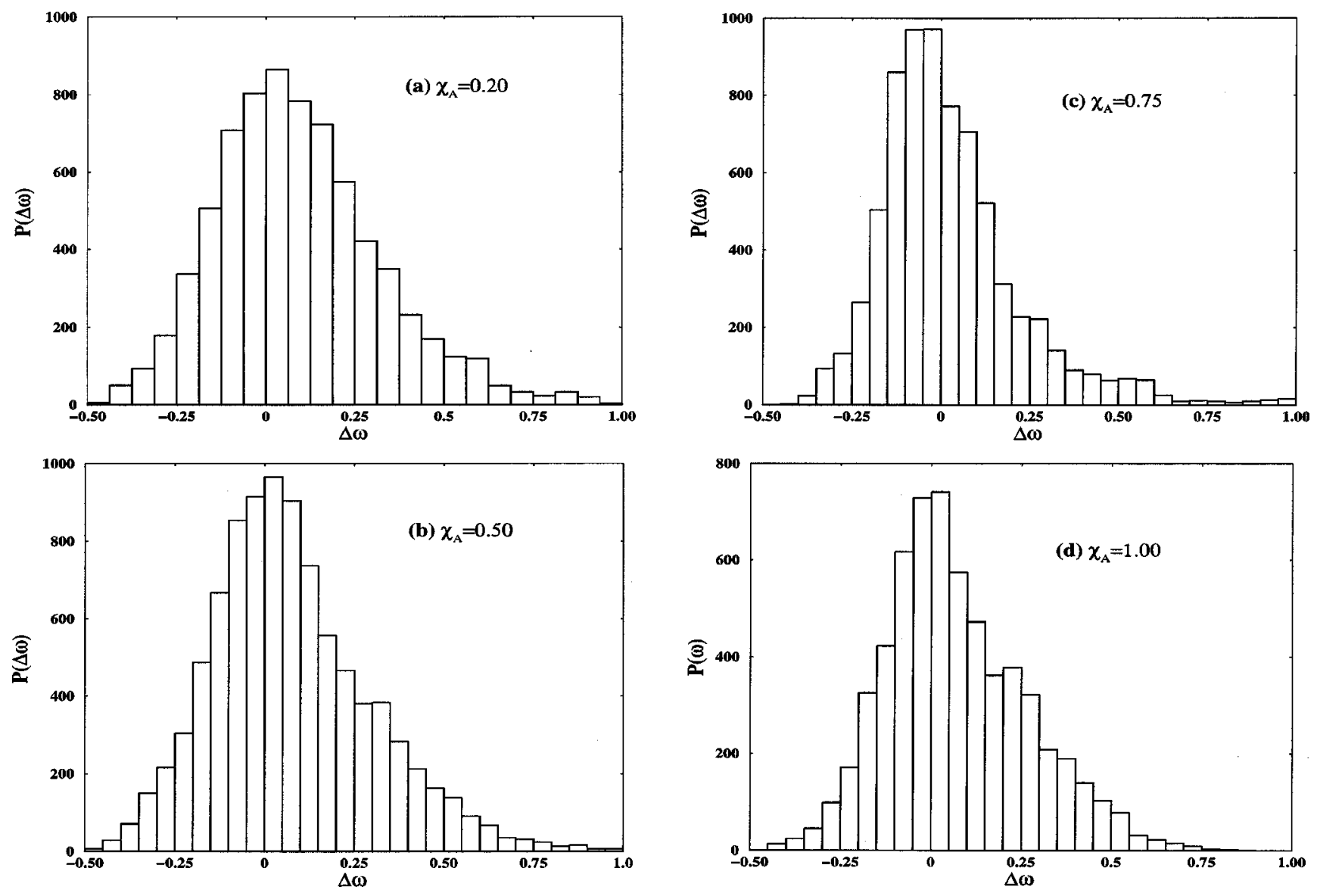

FIG. 6. The probability distribution of the frequency modulation at different compositions, shown in the figure.

is markedly non-Gaussian. The shape is also not Lorentzian and is clearly due to both the slow decay is $C_{\omega}(t)$ and decrease in $\left\langle\Delta \omega^{2}(0)\right\rangle$ at $\chi_{A}=0.5$. We offer a molecular explanation for this behavior later.

Bondarev and Mardaeva ${ }^{11}$ suggested that the band broadening around $\chi=0.5$ in liquid mixtures is due to concentration inhomogeneities. The model of Knapp and Fischer, ${ }^{8,9}$ and Moser et al. ${ }^{20,21}$ assumes the existence of a few environmental states, formed by a discrete number of possible nearest neighbor molecules around the vibrating molecule. Each such state is assumed to cause a specific shift with finite width - the latter is due to transition between different environmental states. Due to the overlap of these frequencies, one broad line should be seen. This means that the line broadening can be attributed to an increase of the amplitude of modulation, i.e., the increase in the mean square frequency modulation. Further, it has been assumed that the influence of diffusional dynamics is small.

However, the simulations presented here lead to somewhat different conclusion. While the mean square frequency modulation $\left\langle\Delta \omega^{2}(0)\right\rangle$ does not show any moderate dependence on the composition (the reason discussed later), the large increase in the correlation time plays a more important role. The latter strongly indicates the importance of slow diffusion exchange dynamics. Muller et al. reported Raman echo experiments on $\mathrm{CH}_{3} \mathrm{I}$ in a $50 \mathrm{~mol} \%$ liquid mixture with $\mathrm{CDCl}_{3}$. They measured a finite lifetime for the concentration fluctuations (4-7 ps). In contradiction with conclusions of Knapp and Fischer, Muller et al. identified the source of the inhomogeneous broadening as due to slow concentration fluctuations in the time domain. This seems to agree with present work.

In order to further understand the origin of nonmonotonic composition dependence, we have computed the the relative contributions of pure and cross correlations to the exponential integrand on the right-hand sight of Eq. (1). The relative contributions are given by the term $X_{i j}(t)$ which is defined as

$$
X_{i j}(t)=\int_{0}^{t} d t^{\prime}\left(t-t^{\prime}\right) C_{\omega}^{i j}\left(t^{\prime}\right) .
$$

In Fig. 7, the time dependent relative contribution of the density [Fig. 7(a)], the vibration-rotation coupling term [Fig. 7(b)], and the cross correlation between them [Fig. 7(c)] are plotted for different compositions. Clearly the density terms make the dominant contributions. Note the sharp rise in the value of the integrand $\left[X_{\rho, \rho}(t), X_{V R, V R}(t)\right]$ near mole fraction 0.5 and the fall when it is crossed. Thus the rise and fall of the dephasing rate arise partly from the rise and fall in the density and the vibration-rotation terms.

The composition dependence of the mean square frequency modulation $\left[\left\langle\Delta \omega^{2}(0)\right\rangle\right]$ is depicted in Fig. 8. This is calculated from unnormalized $C_{\omega}(0)$ at different composition of binary mixture. It is clear that, the variation in $\left\langle\Delta \omega^{2}(0)\right\rangle$ is 

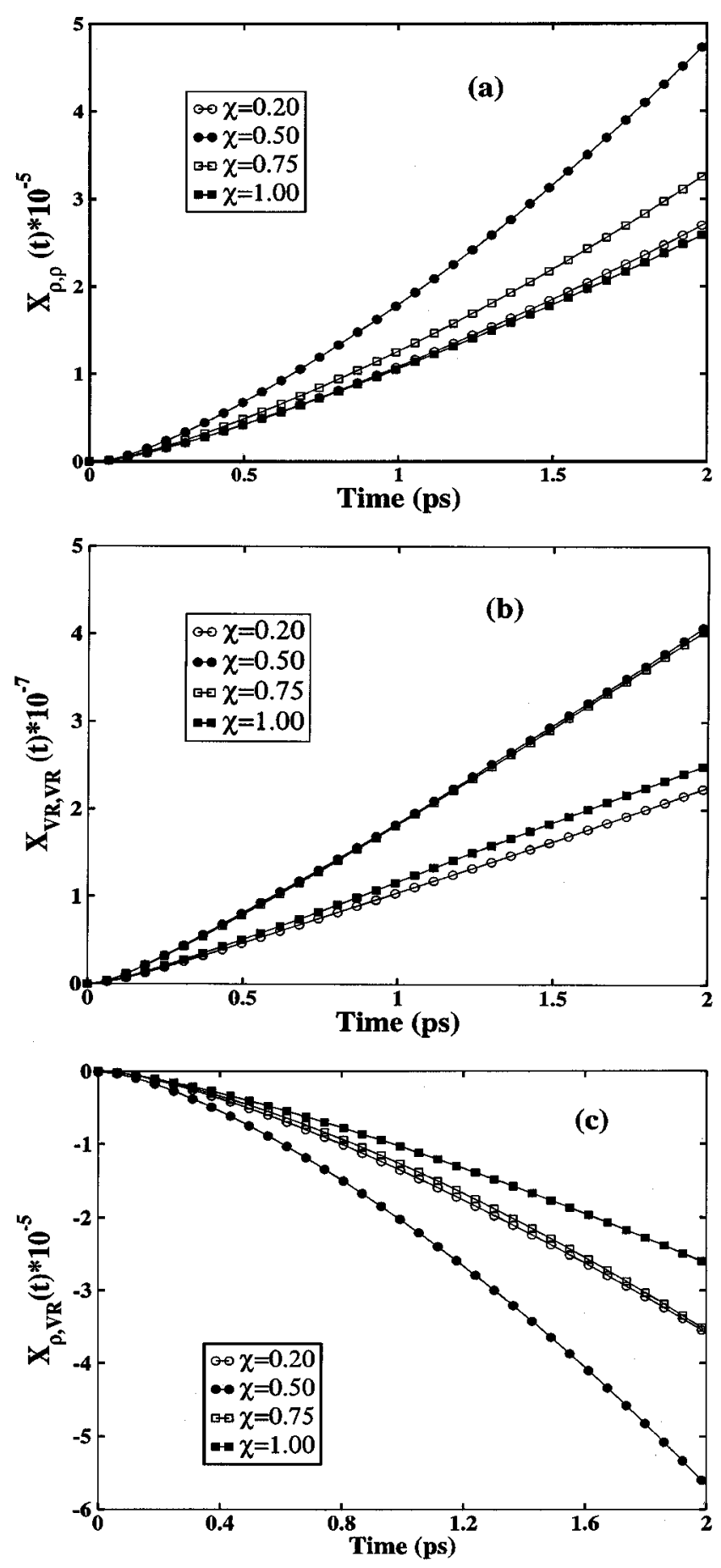

FIG. 7. The time dependence of the relative contributions [to the exponent in Eq. (1)] of the (a) the density-density term $X_{\rho, \rho}(t)$, (b) the VR coupling term $X_{V R, V R}(t)$, and (c) the cross term $X_{\rho, V R}(t)$ between the density and the VR coupling.

small at different composition of binary mixture. As expected, $\left\langle\Delta \omega^{2}(0)\right\rangle$ is minimum at $\chi_{A}=0.5$ because there are large number of each type of species and the composition fluctuation has less effect.

In order to clarify the relative importance of the frequency modulation correlation time in comparison to $\left\langle\Delta \omega^{2}(0)\right\rangle$, we now show the product $\left\langle\Delta \omega^{2}(0)\right\rangle \tau_{c}$ as a function of solute composition $\chi_{A}$ in Fig. 9. The value of $\left\langle\Delta \omega^{2}(0)\right\rangle \tau_{c}$, initially increases with composition and shows a maximum near $\chi_{A}=0.5$ and sharply decreases there after.

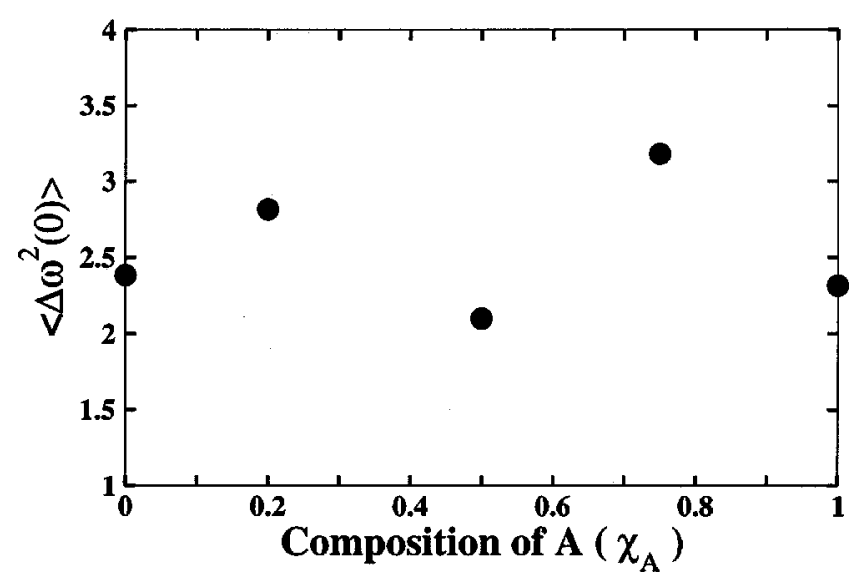

FIG. 8. The mean square frequency modulation $\left(\left\langle\Delta \omega^{2}(0)\right\rangle\right)$ of $A-B$ bond at different compositions of solute $\left(\chi_{A}\right)$.

This nonmonotonic behavior of $\left\langle\Delta \omega^{2}(0)\right\rangle \tau_{c}$ with composition can explain the origin of the nonmonotonicity in the dephasing time (Fig. 3).

The reason for the marked slow down in the correlation time $\tau_{c}$ can be understood by investigating the number density fluctuations for a spherical volume of finite radius. Such a calculation shows that the cross correlation $\left[\left\langle\delta N_{A}(t) \delta N_{B}(0)\right\rangle\right]$ decays much slower than either of the pure terms. ${ }^{22}$ This cross-correlation decay is slowest in the binary mixtures of composition $N_{A} / N=0.5$, because most of the $A$ molecules are surrounded by $B$ molecules. This result clearly suggests that the most slowly relaxing local configurations should involve $A$ molecules surrounded by $B$ molecules which maximizes the number of $A \cdots B$ interactions. This slowness increases the line shape because width is approximately proportional to $\left\langle\Delta \omega^{2}(0)\right\rangle \tau_{c}$.

The above results clearly demonstrate that at least for the present model, the broadening is due to an increase of the correlation time rather than due to increase in the amplitude of modulation, i.e., the mean square frequency modulation. However, a different scenario is also possible.

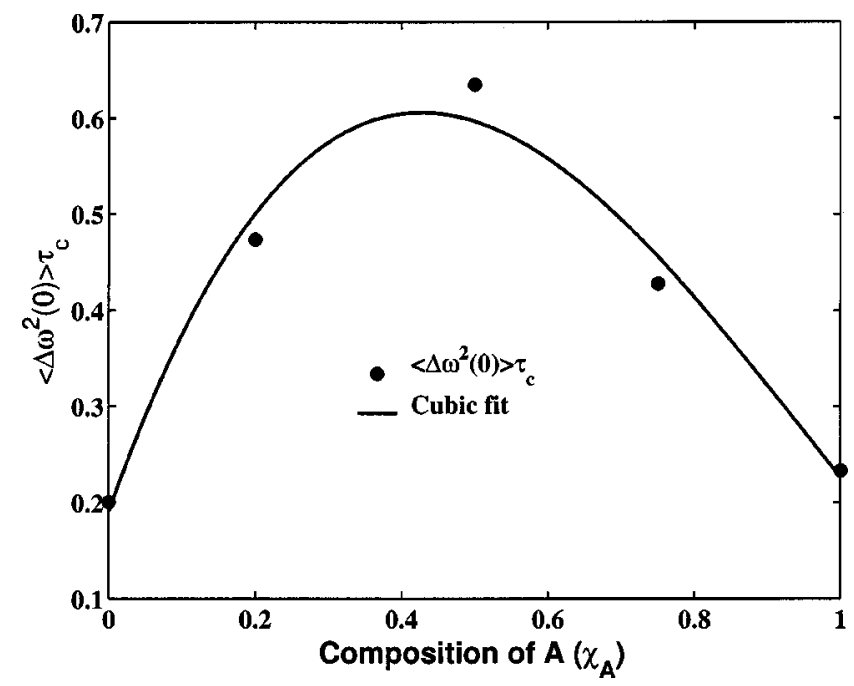

FIG. 9. The value of the product $\left\langle\Delta \omega^{2}(0)\right\rangle \tau_{c}$ at different mole fractions of the solute $\left(\chi_{A}\right)$. We fitted all the points with the third degree polynomial (cubic fit). 


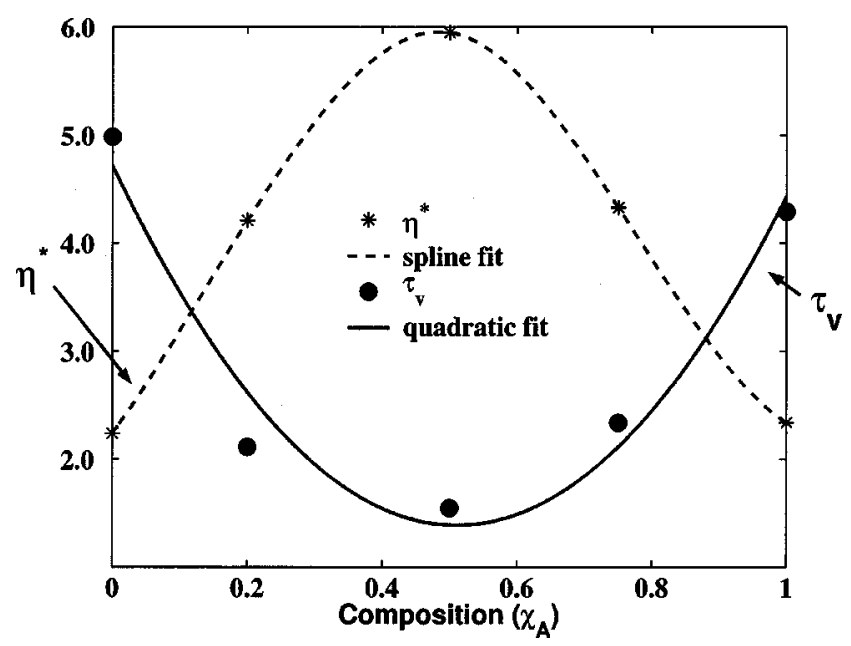

FIG. 10. The calculated average dephasing time (solid circle, $\tau_{v}$ ) and the viscosity $\left(\operatorname{star}, \eta^{*}\right)$ for different mole fractions $\chi_{A}$. The solid line is the quadratic fit for $\tau_{v}$ and the dashed line is the spline fit for $\eta^{*}$.

Numerous studies have found correlation between viscosity and composition dependent dephasing time. ${ }^{1,2}$ In Fig. 10 , we plot the calculated composition dependence of the viscosity $\eta^{*}$ and the dephasing time $\tau_{v}$, respectively. The viscosity has been calculated from the time integral of the stress-stress autocorrelation function and is given by

$$
\eta=\frac{1}{V k_{B} T} \int_{0}^{\infty} d t\left\langle\sigma_{x z}(t) \sigma_{x z}(0)\right\rangle,
$$

where $\sigma_{x z}$ is the off-diagonal element of the stress tensor

$$
\sigma_{x z}=\sum_{j=1}^{N}\left[\left(p_{j}^{x} p_{j}^{z} / m+F_{j}^{z} x_{j}\right] .\right.
$$

Here $F_{j}^{z}$ is the $z$ component of the force acting on the $j$ th particle and the corresponding position of the $j$ th particle is $x_{j}$, and $p_{j}^{z}$ is the $z$ component of the momentum of $j$ th particle, $m$ being the mass of the particle.

In Fig. 10, we compare the composition dependence of $\eta^{*}$ and $\tau_{v}$. Note the striking (and the opposite) nonmonotonic dependence of $\eta^{*}$ and $\tau_{v}$ on solute composition for the two cases. In Fig. 11, we plot $\eta^{*} \tau_{v}$ as a function of composition of $A\left(\chi_{A}\right)$. The product is nearly composition independent.

\section{MODE COUPLING THEORY ANALYSIS}

Mode coupling theory remains the only quantitative and fully microscopic theory for self-diffusion in strongly correlated random systems. In binary mixtures composition fluctuations are obviously expected to play an important role in dephasing akin to the density fluctuations in neat liquid. A mode coupling theory (MCT) analysis have earlier shown that the slow decay of the enhanced density fluctuations near critical point in one component liquid (like $N_{2}$ ) can enhance the rate of dephasing. ${ }^{23,24}$ Oxtoby derived the following expression for the time correlation function of the frequency modulation (due to atom-atom contributions) between two quantum levels $n$ and $m$ in terms of the force-force time correlation function on the atom involved in the bond: ${ }^{1}$

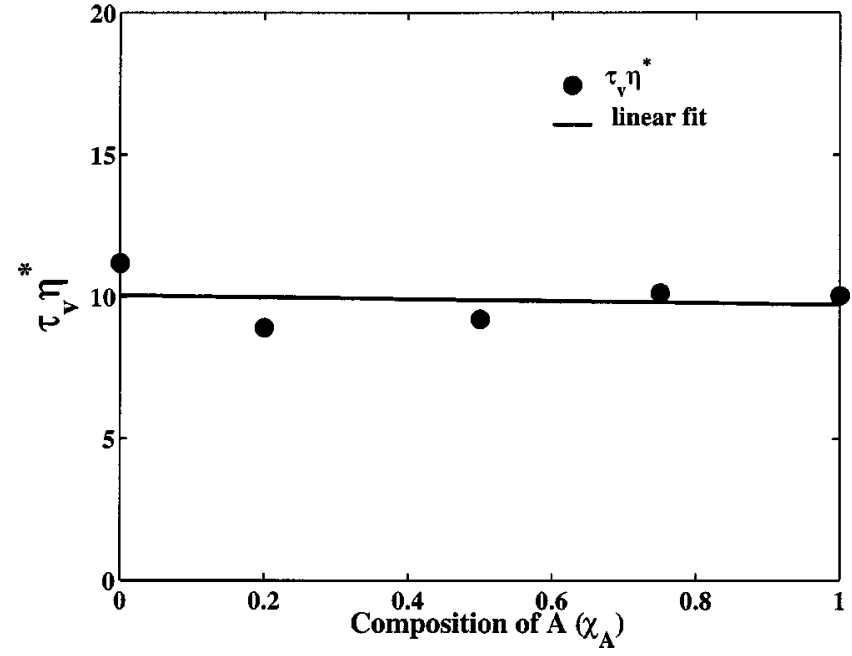

FIG. 11. The values of the product $\tau_{v} \eta^{*}$ at different compositions of solute $\left(\chi_{A}\right)$ in the binary mixture.

$$
\begin{aligned}
\left\langle\Delta \omega_{A A}(t) \Delta \omega_{A A}(0)\right\rangle= & \frac{\left(n^{2}-m^{2}\right)}{2} \sum_{i}\left[\frac{3\left(-k_{111}\right) l_{i k}}{\omega_{0}^{3} m^{1 / 2}}\right. \\
& \left.+\frac{l_{i k}^{2}}{2 \omega_{0} L m_{i}}\right] \times\left\langle\mathbf{F}_{\mathrm{i}}(\mathbf{t}) \mathbf{F}_{\mathrm{i}}(\mathbf{0})\right\rangle,
\end{aligned}
$$

where $L$ is a characteristic potential and $\left\langle\mathbf{F}_{\mathrm{i}}(\mathbf{t}) \mathbf{F}_{\mathrm{i}}(\mathbf{0})\right\rangle$ represents the force-force correlation function [dynamics friction $\zeta(t)]$ (Ref. 12) of the atom $i$ moving along the direction of vibration. For a diatom, $\mathbf{l}_{i k}=\left(m_{i} / \mu\right)^{1 / 2} \gamma_{i}$, where $\gamma_{i}=m_{i} /\left(m_{i}\right.$ $\left.+m_{j}\right) ; m_{i}, m_{j}$ are the mass of $i$ th and $j$ th atom of the diatom and $\mu$ is the reduced mass. The MCT analysis had earlier demonstrated that large enhancement of VR coupling near a gas-liquid critical point could arise from the non-Gaussian behavior of the density fluctuation. ${ }^{13,23,24}$ The density functional theory approach provides the mode coupling theory expression for the force-force time correlation function (see Ref. 13).

The present system differs in many respects from one component systems. Here we have studied a system which is a mixture of two hetero atoms. For a binary mixture, the mode coupling theory analysis gives the following expression for the force-force time correlation function:

$$
\begin{aligned}
\left\langle F^{i}(t) F^{j}(t)\right\rangle= & \frac{k_{B} T}{6 \pi^{2}} \int_{0}^{\infty} d \mathbf{k} k^{4}\left[F_{s}^{i j}(\mathbf{k}, t)-F_{s 0}^{i j}(\mathbf{k}, t)\right] \\
& \times\left[\mathbf{C} * \mathbf{F} * \mathbf{C}^{\dagger}\right]_{i j},
\end{aligned}
$$

where $F_{s}^{i j}(t)=\left\langle n_{i}(t) n_{j}(t)\right\rangle$ is the self-intermediate scattering function of the solute and $F_{s 0}^{i j}(\mathbf{k}, t)$ is the inertial part of the $F_{s}^{i j}(\mathbf{k}, t)$. The direct correlation matrix $\mathbf{C}$ and the intermediate scattering function $\mathbf{F}$ are expressed as

$$
\mathbf{C}=\left(\begin{array}{ll}
C_{11} & C_{12} \\
C_{21} & C_{22}
\end{array}\right), \quad \mathbf{F}(\mathbf{k}, \mathbf{t})=\left(\begin{array}{ll}
F_{11}(k, t) & F_{12}(k, t) \\
F_{21}(k, t) & F_{22}(k, t)
\end{array}\right) .
$$

To calculate the friction, the recently proposed generalized self-consistent scheme can be used, which makes use of the well-known Gaussian approximation for $F_{s}(k, t){ }^{25,26}$ Such a calculation has been carried out earlier for binary 
mixture, for two models of nonideal fluids, both having interaction potentials similar to the ones chosen here. Therefore, these calculations can be used to derive insight regarding vibrational dephasing in the present system. The friction on the individual atoms show strong bimodal response in $\langle F(t) F(0)\rangle$ - a Gaussian behavior in the initial time scale followed by a slowly relaxing component. There is even a rise in friction in the intermediate time scale. This arises from the coupling of the solute motion to the collective density relaxation of the solvent. ${ }^{26}$ The Gaussian component arises from isolated binary interactions and the slower part arises from the correlated recollisions. However, the frictions on the two dissimilar parts are found to be quite different.

In the mode coupling theory calculation for the vibrational dephasing in the one component system $\mathrm{CH}_{3} \mathrm{I}$, the friction was found to be much higher in the case of I than that of $\mathrm{CH}_{3}$. This is expected because although the LJ diameters of $\mathrm{CH}_{3}$ and I spheres are nearly equal, their individual masses are considerably different $\left(\mathrm{CH}_{3}=15 \mathrm{~g} / \mathrm{mol}\right.$ and $\mathrm{I}=126 \mathrm{~g} / \mathrm{mol}$ ). Because of this large mass disparity, the positional coordinate on the bond corresponding to the equilibrium point would be much closer to the iodine atom. As a result, the iodine atom is a lot more static than $\mathrm{CH}_{3}$. The large value of the friction for iodine also arises from its larger $\epsilon$ value. The mass term effects not only in the friction but also in the frequency modulation time correlation function [Eq. (11)]. Since the mass is in the denominator of the prefactor of Eq. (11), this further reduces the contribution of the heavy atom. Details of the calculations will be published elsewhere. Such a result is also expected in the present problem. Detailed numerical calculation is in progress.

Mode coupling theory is known to be quite successful in estimating the viscosity of dense liquids below the glass transition temperature. The mode coupling contribution to viscosity can be evaluated by using the general approach initiated by Bosse et $a{ }^{27}$ and further developed by Gestzi. ${ }^{28}$ In this approach one starts with the general time correlation function expression for the shear viscosity in terms of the transverse current. For a binary mixture, the final expression for the mode coupling part of viscosity can be obtained by following the method outlined in Refs. 27 and 28 and is given by ${ }^{12}$

$$
\begin{aligned}
\eta & =\sum_{i, j=1}^{2} \eta_{\rho_{i} \rho_{j}} \\
& =\sum_{i, j=1}^{2} \frac{k_{B} T}{60 \pi^{2}} \int_{0}^{\infty} d q q^{4} \frac{S_{i i}^{\prime}(q) S_{j j}^{\prime}(q)}{S_{i i}^{2}(q) S_{j j}^{2}(q)} \int_{0}^{\infty} d t F_{i j}^{2}(q, t),
\end{aligned}
$$

where $\rho_{i}$ is the number density of the $i$ th species ( $A$ and $B$ ). $S_{i i}(q)$ and $S_{i i}^{\prime}(q)$ are the static structure factor and its derivative for the $i$ th species. It is instructive to note the similarity between Eqs. (12) and (13) — both involve integration over the intermediate scattering function matrix $\mathbf{F}$. It is the latter which contains effects of composition fluctuation. In the above expressions, the dynamical input parameters are the partial intermediate scattering functions $F_{i j}(q, t)$. Using the above Eq. (13), Srinivas et al. have successfully explained the nonmonotonic composition dependence of the viscosity in the binary mixture. ${ }^{12}$ They also have compared MCT predictions with molecular dynamics simulation results.

The close similarity in the composition dependence between the vibrational dephasing time and the viscosity finds an elegant explanation in the mode coupling theory in terms of the intermediate time scattering function. Thus, two rather different relaxation processes (phase and stress) are dominated by the composition fluctuation in binary mixture.

\section{CONCLUSION}

Understanding the nonmonotonic vibrational phase relaxation in a binary mixture is indeed a challenging problem. In addition to the inherent complexity of studying vibrational phase relaxation in dense liquids (multiple contributions), the contribution due to composition fluctuation gives rise to additional complexity. While this problem has attracted theoretical studies before, we are not aware of any computer simulation study of all the terms and cross terms that contribute to phase relaxation.

The present simulations have been successful in reproducing several aspects of the experimentally observed results, especially the nonmonotonic composition dependence. The composition dependence of the vibrational phase relaxation time is found to be quite symmetric with the maximum located close to $\chi_{A}=0.5$. The value at the maximum is about three times larger than that for the pure components. Analysis of the various terms contributing to the frequency modulation time correlation function demonstrates that this increase is because of the slow down of density relaxation terms near the said composition. Earlier studies of a similar system demonstrated that this slow down in composition relaxation is due to the contribution of the cross-terms $\left[\left\langle\delta N_{A}(t) \delta N_{B}(0)\right\rangle\right]$. A somewhat surprising result is the relative insensitivity of the mean square frequency term to the variation in composition.

We have calculated the variation of viscosity with the composition and found that this also exhibits a nonmonotonic composition dependence. In fact, the correlation between viscosity and the dephasing time is rather striking in the present model. Mode coupling theory is known to provide an elegant explanation of the composition dependence of viscosity. We have presented the basic MCT equations here and analyzed them in qualitative terms, by using earlier calculations. A full MCT calculation of the present problem is under progress.

The close resemblance in the composition dependence of the dephasing rate with that of the viscosity might be due to the specific features of the model employed, but it suggests that experiments should certainly compare the two dependencies, as a lot can be learned by such comparison. In addition, one needs to look at the temperature dependence of the dephasing rate in binary mixtures. At higher temperatures, the contribution of vibration-rotation mechanism is expected to increase, which may weaken the close correlation between the dephasing rate and the viscosity. Another worthwhile future problem is to simulate $\mathrm{CDCl}_{3}$ and $\mathrm{CH}_{3} \mathrm{I}$ binary mixture using realistic potential. 


\section{ACKNOWLEDGMENTS}

The authors thank Dr. S. Bhattacharyya, A. Mukherjee, and P. P. Jose for helpful discussions. S.R. acknowledges the CSIR (India) for financial support. This work was supported in part by grants from Department of Science and Technology, India and Council of Scientific and Industrial Research, India.

${ }^{1}$ D. W. Oxtoby, Adv. Chem. Phys. 40, 1 (1979); D. W. Oxtoby, J. Chem. Phys. 70, 2605 (1979).

${ }^{2}$ D. Levesque, J. J. Weis, and D. W. Oxtoby, J. Chem. Phys. 72, 2744 (1980).

${ }^{3}$ R. Kubo, Adv. Chem. Phys. 15, 101 (1969).

${ }^{4}$ H. T. Lotz, J. P. J. Michels, and J. A. Schouten, J. Chem. Phys. 117, 7245 (2002).

${ }^{5}$ F. Lindenberger, R. Stockl, B. P. Asthana, and A. Laubereau, J. Phys Chem. A 103, 5655 (1999).

${ }^{6}$ L. J. Muller, D. V. Bout, and M. Berg, J. Chem. Phys. 99, 810 (1993).

${ }^{7}$ B. P. Asthana and W. Kiefer, Vibrational Spectra and Structure, edited by

J. R. Durig (Elsevier, Amsterdam, 1993), Vol. 20, p. 67.

${ }^{8}$ E. W. Knapp and S. F. Fischer, J. Chem. Phys. 74, 89 (1981).

${ }^{9}$ E. W. Knapp and S. F. Fischer, J. Chem. Phys. 76, 4730 (1982).
${ }^{10}$ B. P. Asthana, H. Takahashi, and W. Kiefer, Chem. Phys. Lett. 94, 41 (1983).

${ }^{11}$ A. F. Bondarev and A. I. Mardaeva, Opt. Spektrosk. 35, 286 (1973).

${ }^{12}$ A. Mukherjee, G. Srinivas, and B. Bagchi, J. Chem. Phys. 114, 6220 (2001).

${ }^{13}$ N. Gayathri, S. Bhattacharya, and B. Bagchi, J. Chem. Phys. 107, 10381 (1997).

${ }^{14}$ W. G. Hoover, Phys. Rev. A 31, 1695 (1985)

${ }^{15}$ S. Nose, Mol. Phys. 52, 255 (1984).

${ }^{16}$ H. C. Andersen, J. Chem. Phys. 72, 2384 (1980).

${ }^{17}$ G. J. Martyna, D. J. Tobias, and M. L. Klein, J. Chem. Phys. 101, 4177 (1994).

${ }^{18}$ K. F. Everitt and J. L. Skinner, J. Chem. Phys. 115, 8531 (2001).

${ }^{19}$ R. M. Lynden-Bell and J. C. Rasaiah, J. Chem. Phys. 107, 1981 (1997).

${ }^{20}$ G. Moser, A. Asenbaum, J. Barton, and G. Doge, J. Chem. Phys. 102, 1173 (1995).

${ }^{21}$ J. Chesnoy and G. M. Gale, Adv. Chem. Phys. 70, 299 (1988).

${ }^{22}$ R. K. Murarka and B. Bagchi, J. Chem. Phys. 117, 1155 (2002).

${ }^{23}$ S. Roychowdhury and B. Bagchi, J. Chem. Phys. 119, 3278 (2003).

${ }^{24}$ S. Roychowdhury and B. Bagchi, Phys. Rev. Lett. 90, 075701 (2003)

${ }^{25} \mathrm{U}$. Balucani and M. Zoppi, Dynamics of the Liquid State (Clarendon, Oxford, 1994).

${ }^{26}$ B. Bagchi and S. Bhattacharyya, Adv. Chem. Phys. 116, 67 (2001).

${ }^{27}$ J. Bosse, W. Gotze, and M. Lucke, Phys. Rev. A 17, 434 (1978).

${ }^{28}$ T. Geszti, J. Phys. C 16, 5805 (1983). 